XXII.

Aus der III. medicinischen Klinik in Budapest.

(Director: Prof. Baron A. v. Korányi.)

\title{
Ueber den Einfluss des Pankreas-Presssaftes auf den Blutkreislauf.
}

Von

Privatdocent Dr. R. Bálint und Dr. B. Molnár.

(Mit 1 Curre im Text.)

Wir haben in unseren früheren Versuchen (Berl. klin. Wochenschr. 1911. No. 7) gefunden, dass der Thyreoideapresssaft in gewissen Beziehungen sich dem Adrenalin ähnlich verhält, indem es beim Versuchsthier den Eintritt der Strychninwirkung zu hemmen im Stande ist. Dagegen paralysirt der Pankreaspresssaft die Antistrychninwirkung, so des Adrenalins wie des Thyreoideapresssaftes. In unseren Nitroglycerinversuchen haben wir nachgewiesen, dass die Ursache der Antistrychninwirkung des Adrenalins und des Thyreoideapresssaftes in ihrer vasoconstrictorischen Wirkung zu suchen ist. Auf Grund der Aehnlichkeit der Wirkungen dachten wir annehmen zu können, dass sowohl der Pankreaspresssaft als das Nitroglycerin die vasoconstrictorische Wirkung des Adrenalins und 'Thyreoideapresssaltes paralysirt, das heisst, dass der Pankreaspresssaft als Antagonist des Adrenalins und des Thyreoideapresssaftes vasodilatirend wirkt. Zur genaueren Klärung dieser Frage haben wir mit den Presssäften des Pankreas und anderer Organe in Bezug auf ihre Gefässwirkung ausführliche Untersuchungen angestellt. DerartigeUntersuchungen liegen zwar bezüglich einzelner Organe in grösserer, anderer Organe in kleinerer Zahl in der Literatur vor, dennoch erschien uns eine ausführlichere Bearbeitung dieser Frage speciell um so lohnenswerther, da unsere früheren Versuche eine ausgesprochene Gefässwirkung des Pankreaspresssaftes demonstriren. Die in der Literatur mitgetheilten Versuche weisen übrigens grösstentheils mehrere Mängel auf. Als einen solchen müssen. wir unter anderem bezeichnen, dass die Blutdruckversuche grösstentheils mit Quecksilbermanometer angestellt wurden, womit die Blutdrucksteigerung und Senkung zwar in Zahlen ausgedrückt werden kann, aber die graphische Darstellung der Blutdruckschwankungen nur unvollkommen ausgeführt werden kann, da theils die Selbstschwingungen des Quecksilbers die graphische Curve modificiren, theils aber die ziemlich grosse Quecksilbersäule die feineren Schwingungen um so woniger treu zurückgeben kann, da diese Blutdruckmessungen an Kaninchen aus- 
geführt wurden und der auch absolut kleine Blutdruck dieses Versuchsthieres sich zur graphischen Darstellung wenig eignet.

Aus diesen Gründen zeigte ein grosser Theil dieser Versuche nur die Aenderung des Blutdruckes meistens ohne die Entscheidung der Frage zu ermöglichen, ob die Blutdruckänderung central, ob sie peripher bedingt sei. Wir stellten unsere Versuche am Hunde an. Die grosse Wichtigkeit der graphischen Darstellung vor Augen haltend, haben wir den Apparat von Hürthle-Gad benutzt, wo das Blut durch eine in die Carotis gebundene Canüle und durch Vermittlung einer $\mathrm{MgSO}_{4}$-Flüssigkeitssäule eine feine Gummimembran in Bewegung stetzt, deren Bewegung durch einen feinen Schreiber auf Russpapier registrirt wird. Ein Vorzug dieses Verfahrens besteht jedenfalls darin, dass die aufgezeichnete Curve den kleinsten Schwankungen des Blutdruckes folgt. Dagegen ist ein allerdings unbedeutender Nachtheil unserer Versuche, dass der Blutdruck in absoluten Zahlen nur nach indirecten Messungen ausgedrückt werden kann. Die Presssäfte wurden von ganz frischen Organen durch Pressen unter hohem Druck gewonnen und intravenös angewendet. Den Hund hielten wir in Morphiumbetäubung.

Wir theilen nun unsere Versuche nach den einzelnen Presssäften gruppirt kurz in Folgendem mit.

Nach intravenöser Einführung von Pankreaspresssaft verändert sich die Blutdruckcurve nach einigen Secunden mit einer geradezu gesetzmässigen Consequenz. Die Blutdruckcurve fällt plötzlich in steilem Abhang herab, inzwischen wachsen die einzelnen Schwingungen mächtig an, die ansteigenden und abfallenden Theile der Curve werden auffallend steil, bilden oben eine scharfe Spitze und es zeigt sich eine hochgradige Dikrotie. In der Pulsfrequenz haben wir keine gesetzmässige Veränderung gefunden, indem wir in einzelnen Fällen den Eintritt einer ganz geringfügigen Tachykardie, in anderen dagegen einer kurzdauernden geringen Bradykardie beobachtet haben. Der Pankreaspresssaft übt also eine ganz constante, geradezu gesetzmässige Wirkung auf das Gefässsystem aus, indem der Blutdruck sinkt, die systolischen Erhebungen mächtig anwachsen, ein Hyperdicrotismus entsteht, der auf eine hochgradige Entspannung der Gefässwand hindeutet. Ein charakteristisches Beispiel der Pankreascurven zeigt die mitgetheilte Figur, welche eine Curve unserer zahlreichen, bei ähnlichen Versuchen gewonnenen darstellt.

Diese biutdruckherabsetzende Wirkung des Pankreaspresssaftes hält nicht lange an; die verschiedenen Presssäfte und Thierindividuen verhalten sich diesbezüglich bis zu einem Grade individuell verschieden. Im Allgemeinen können wir aber sagen, dass die, durch den Pankreaspresssaft verursachte Blutdrucksenkung nur einige Minuten (gewöhnlich 4-5 Min.) dauert. Später steigt der Blutdruck wieder an, während die ansteigenden und fallenden Curventheile weniger steil werden, der Dicrotismus verschwindet und die Pulscurve wieder normal wird. Nach erneuerter Injection des Saftes können wir die geschilderte charakteristische Wirkung wieder beobachten, die etwa noch vorhandene wird gesteigert. Die nach der Einführung des Pankreaspresssaftes eintretende Senkung des Blutdruckes ist, soweit dies aus der Form der Curven ermittelt werden kann, die Folge 
der Erschlaffung der Gefässe. Das plötzliche Anwachsen der systolischen Erhebungen bei gleichzeitigem Sinken des Blutdruckes kann nur durch die Entspannung der Gefässwände hervorgebracht werden. Die Aenderung der Herzfunktion spielt in diesem Falle bei der Blutdrucksenkung keine Rolle, dies wird eclatant durch unsere Versuche demonstrirt, die wir bei gleichzeitiger Einführung des Pankreaspresssaftes mit dem in die $\mathrm{Ca}$ rotis des Thieres eingebundenen Vierordt'schen Tachometer angestellt haben. Der Tachometer zeigte nämlich in jedem Falle eine Zunahme der Geschwindigkeit des Blutkreislaufes. In einem Falle war die Geschwindigkeitszunahme sehr beträchtlich, indem der Zeiger des Tachometers einen Ausschlag von 2,5 auf 4 aufwies. Diese Zunahme der Geschwindigkeit des Blutstromes bei gleichzeitiger Senkung des Blutdruckes spricht ohne Zweifel gegen den cardialen Ursprung der Senkung. Wie wir sehen werden, steht diese Wirkung des Pankreaspresssaftes in scharfem Gegensatz zu der Wirkung des thyreoiden Presssaftes. Nachdem wir uns davon überzeugt haben, dass die Wirkung des Pankreaspressaftes auf den Blutdruck nicht durch unmittelbare Beeinflussung des Herzens entsteht, war zu entscheiden, ob die durch Pankreaspressaft verursachte Gefässentspannung infolge der Lähmung des vasomotorischen Centrums entstehe, oder aber die Folge peripherer Wirkung sei. Die nach Durchschneidung des Rückenmarkes und bei künstlicher Athmung angestellten PankreaspresssaftVersuche zeigten, dass die gefässentspannende Wirkung des Pankreaspresssaftes auch nach der Ausschaltung der vasomotorischen Centren, also von ihnen unabhängig zu Stande kommt. Folglich wirkt der Pankreaspresssaft an der Peripherie

In der technischen Ausführung dieser Versuche hielten wir uns an bekannte experimental-physiologische Methoden. Am morphinisirten Thiere führten wir die Tracheotomie aus und die in die Trachea eingeführte Canüle haben wir mit dem Mayer'schen, zur künstlichen Athmung dienenden Apparat in Verbindung gebracht, nachher haben wir zwischen dem Occiput und dem ersten Wirbel nach gehörigem Auspräpariren das Rückenmark durchschnitten, worauf die Reizung des peripheren Rückenmarktheiles möglich wurde. Für eine periphere Wirkung sprachen übrigens auch unsere, an curarisirten Hunden gemachten Beobachtungen. Nach der Injection einer gewissen Quantität von Curare entwickelte sich nämlich die gefässentspannende Wirkung des eingeführten Pankreaspresssaftes ungestört, doch blieb nach Injection

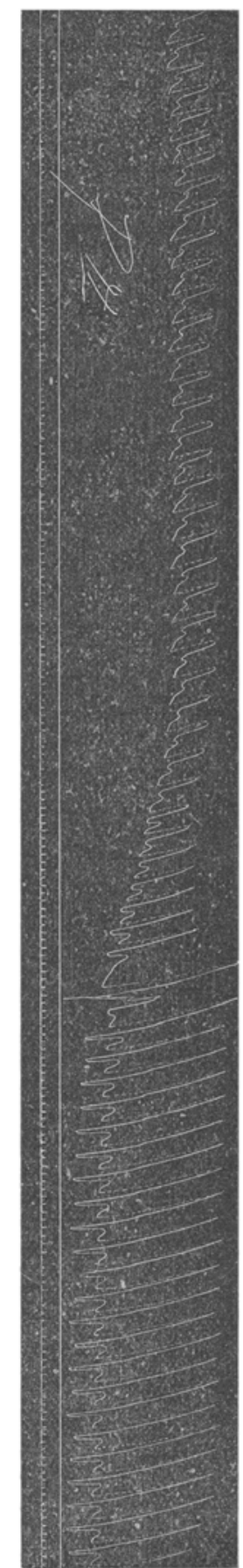


von grösseren Mengen Curare die Pankreaspresssaft-Wirkung aus. Diese Beobachtung lässt kaum eine andere Erklärung zu, als die Annahme, dass die durch Curare in grossen Dosen hervorgerufene maximale Vasodilatation eine zweite Zunahme der Gefässe unmöglich macht.

Nach Vagusdurchschneidung oder beim atropinisirten Thiere kommt die gefässentspannende Wirkung des Pankreaspresssaftes prompt zu Stande. Diesbezüglich zeigt sich also ein scharfer Gegensatz zwischen der Wirkung des Cholins und des Pankreaspresssaftes. Wie ältere und besonders neuestens die Versuche von Abderhalden und Müller zeigten, setzen die wirksamen Dosen von Cholin den Blutdruck herab. Dieser herabgesetzte Blutdruck steigt aber wieder an, wenn wir den Vagus durchschneiden, oder wenn wir das Thier atropinisiren. Das Cholin übt seine Wirkung also auf dem Wege des Vagus aus, worauf wir übrigens noch später zurückzukommen haben werden, während der Pankreaspresssaft seine gefässentspannende Wirkung auch bei gelähmten Vagi ausübt.

Die äussere Form der Gefässwirkung des Pankreaspresssaftes steht in vollem Gegensatz zur Gefässwirkung des Adrenalins. Das Adrenalin bringt Vasoconstriction und Blutdrucksteigerung, der Pankreaspresssaft Vasodilatation und Blutdrucksenkung hervor. Die äusseren Umstände der Wirkungen dieser beiden Substanzen sind einander aber sehr ähnlich. Bei beiden stellt sich die Wirkung plötzlich ein, erreicht plötzlich ihren Höhepunkt und verschwindet auch schnell. Eine gewisse Aehnlichkeit zeigt sich auch darin, dass beide an der Peripherie angreifen, obzwar wir kaum einen ganz sicheren Beweis dafür erbringen konnten, dass der Pankreaspresssaft ähnlich wie das Adrenalin direct auf die Gefässwände wirkt.

Bei gleichzeitiger Injection von Adrenalin und Pankreaspresssaft in entsprechenden Dosen compensiren sich die entgegengesetzten Wirkungen vollkommen, während in Versuchen, in welchen die relativen Mengen des Adrenalins und des Pankreaspresssaftes andere waren, bald eine Erhöhung, bald eine Erniedrigung des Blutdruckes vorherrschte.

Die Gefässwirkung des Pankreaspresssaftes könnte verschiedenen Körpern zugeschrieben werden. Von solchen kommen vor allem das Pepton und Cholin in Betracht.

Die Untersuchungen von Schmidt und Mühlheim zeigten nämlich, dass das Pepton eine gefässentspannende Wirkung hat. Diese Wirkung des Peptons ist übrigens vorläufig nicht geklärt. Einzelne haben vom reinen Pepton keine Gefässwirkung gesehen und schreiben diese Wirkung den, dem Pepton beigemengten Substanzen (das Peptozym von Pick und Spiro) zu. Popielski nennt diese Substanz Vasodilatin und hält sie für identisch mit Substanzen, die aus verschiedenen Organen (Leber, Milz, Pankreas etc.) gewinnbar sind. Letztere Annahme konnte zu dem Gedanken verleiten, dass die vasodilatorische Wirkung des Pankreaspresssaftes etwa dem Pepton zuzuschreiben sei. Zur Klärung dieser Frage haben wir vor allem Blutdruckversuche mit verschiedenen Peptonen ausgeführt. Die Versuche zeigten, dass das Pepton siccum sine sale Merck den Blutdruck unbeeinflusst liess. Das Pepton Witte und das Pepton e carne Merck verursachten zwar Blutdrucksenkung, aber die von der 
Carotis aufgenommenen Blutdruckcurven waren der Blutdruckcurve des Pankreaspresssaftes überhaupt nicht ähnlich, indem die Zunahme der systolischen Erhebungen als Zeichen einer Entspannung der Gefässe nicht zu betrachten war. Unser Befund deckt sich übrigens vollkommen mit den Untersuchungsergebnissen von Thompson, der gefunden hat, dass die durch das Pepton verursachte Gefässentspannung hauptsächlich in den zum Splanchnicusgebiet gehörenden Gefässen zu constatiren ist. Die gefässentspannende Wirkung des Pankreaspresssaftes kann daher auch von diesem Standpunkte aus mit Peptonwirkung nicht für identisch gehalten werden. Andererseits konnten wir in den injicirten und sich wirkungsvoll zeigenden Presssäften Pepton chemisch nicht nachweisen.

Die andere Substanz, die in Frage kommen könnte, war das Cholin. Dass aber die Wirkung des Pankreaspresssaftes nicht mit der Cholinwirkung identisch sein kann, das illustrirt schon die früher erwähnte Thatsache, dass eine Cholinwirkung nur bei Vagusfunction zu Stande kommen kann, und nach Durchschneidung oder Atropinisirung der Vagi aufhört und einer Blutdrucksteigerung Platz macht, während die blutdrucksenkende Wirkung des Pankreaspresssaftes von der Function der Vagi unabhängig ist und bei Hunden mit durchschnittenen oder atropinisirten Vagi, ebenso zu Stande kommt, wie bei denen mit intacten Vagi. Die Conclusion, die sich aus diesen Versuchen ergiebt, ist, dass die gefässentspannende Wirkung des Pankreaspresssaftes von der blutdrucksenkenden Wirkung des Cholins sich principiell unterscheidet.

Es konnte noch in Frage kommen, ob bei der blutdrucksenkenden Wirkung des Pankreaspresssaltes nicht Eiweissspaltungsproducte eine Rolle spielen. Das könnte man um so mehr supponiren, da auf die Einwirkung des im Pankreaspresssaft vorhandenen Trypsins solche Spaltungsproducte entstehen könnten. Wir haben zwar im Pankreaspresssaft actives Trypsin nie gefunden, indem die Mett'schen Röhrchen unverdaut blieben, aber wir wollten der Frage auch in einer anderen Richtung näher treten; wir versetzten Leberpresssaft mit Trypsin, liessen diese Mischung durch mehrere Stunden stehen, und nach Aufkochen injicirten wir es in das Versuchsthier. Diese Untersuchungen zeigten, dass in der Wirkung des einfachen Leberpresssaftes und des mit Trypsin versetzten, kein Unterschied besteht. Dieser Befund beweist auch, dass die Ursache der vasodilatirenden Wirkung des Pankreaspresssaftes nicht in Eiweissspaltungsproducten zu suchen ist.

Der Pankreaspresssaft behält diese gefässentspannende Wirkung auch nach Aufkochen und auch dann, wenn behufs Enteiweissung der Presssaft mehrmals mit Alkohol behandelt und nachher mit Wasser diluirt wird.

Nachdem die Gefässwirkung des Pankreaspresssaftes weder dem Cholin noch dem Pepton zugeschrieben werden kann, ist die Annahme berechtigt, dass sie einem, dem Pankreas specifisch zukommenden Stoffe ihren Ursprung verdankt. Die Berechtigung dieser Annahme hängt von den Ergebnissen von Untersuchungen ab, welche zu entscheiden haben, ob die Gefässwirkung des Pankreaspresssaftes sich von den anderen Presssäften principiell unterscheidet oder nicht. In der That haben z.B. Vincent, Miller eine gefässentspannende Wirkung für Presssäfte mehrerer 
Organe festgestellt. Zur Klärung dieser Frage hielten wir vor allem für nothwendig, Presssäfte verschiedener Organe zu untersuchen. Unter diesen Organen musste zuerst die Thyreoidea in Betracht kommen, theils deshalb, weil die bisherigen Forscher fast ausnahmslos gefunden haben, dass der Thyreoideapresssaft den Blutdruck herabsetzt, theils aber auch deshalb, weil wir in unserer oben citirten und erschienenen Arbeit gefunden haben, dass in den Strychninversuchen die Wirkungen des Thyreoideapresssaftes und des Adrenalins einander ähnlich sind.

Nach der Injection des Thyreoideapresssaftes sinkt thatsächlich der Blutdruck des Versuchsthieres, aber die Blutdruckcurve unterscheidet sich prineipiell von der Blutdruckcurve, die nach der Injection von Pankreaspresssaft entsteht. Die Senkung des Blutdruckes tritt kurz nach der Injection ein, die Curve fällt allmählich tiefer, gleichzeitig werden aber die systolischen Ausschläge des registrirenden Apparates nicht grösser wie in unserem Pankreasversuche, sondern einem gespannten Pulse entsprechend kleiner. An den Curven werden die Spitzen durch kleine Plateaux ersetzt und die etwa früher vorhandenen dikrotischen Erhebungen bleiben aus. Ausgesprochene Elasticitätsschwingungen treten auf. Nach dem Abklingen der blutdrueksenkenden Wirkung erhebt sich der Blutdruck wieder, aber die Curve behält noch längere Zeit ihre, einem gespannten Pulse entsprechende Form. Es kamen nach Thyreoideapresssaftinjection auch solche Curven zur Beobachtung, in welchen Blutdrucksenkung überhaupt nicht zu constatiren war und nur die Verminderung der systolischen Erhebungen zu erkennen war. Die aufgenommenen Curven zeigten also, dass nach der Injection von Thyreoideapresssaft sich die peripheren Gefässe contrahiren, was auch unserem, schon früher erwähnten experimentellen Befund entspricht, nach welchen der Thyreoideapresssaft sich in den Strychninversuchen dem Adrenalin ähnlich verhält. Vielleicht könnte man daran denken, dass die bei den Thyreoideathieren erhaltenen Curven einer Vasodilatation im Splanchnicusgebiete zugeschrieben werden können, was einerseits die Blutdrucksenkung, andererseits die compensatorische Contraction der peripheren Gefässe erklären würde. Dass das aber nicht der Fall ist, das zeigten unsere Versuche, welche wir nach der Thyreoideapresssaftinjection mit dem Vierordt'schen Tachometer ausführten. Diese Untersuchungen zeigten, dass nach der Thyreoideapressaftinjection bei der Senkung des Blutdruckes eine wesentliche Verlangsamung des Blatkreislaufes zu constatiren ist; so fiel in einem unserer Versuche der Zeiger des Tachometers von 2,5 auf 0,5 herab, wobei der Blutdruck eine wesentliche Senkung zeigte. Diese experimentellen Ergebnisse weisen also darauf hin, dass wir die Ursache der Blutdrucksenkung in einer Aenderung der Herzfunction zu suchen haben, entsprechend dem Befunde von Haskovecz, der gefunden hat, dass die Thyreoidea eine cardiale Hypotonie verursacht. Diese cardiale Hypotonie kann durch die Contraction peripherer Gefässe in einzelnen Fällen in ihrer Wirkung auf den Blutdruck compensirt werden; dies scheinen auch jene Curven zu demonstriren, bei welchen der Thyreoideapresssaft keine Blatdrucksenkung hervorruft und nur das Kleinerwerden der systolischen Erhebungen und einen gespannten 
Puls verursacht. In den meisten Fällen aber gelingt die völlige Compensation nicht und der Blutdruck sinkt trotz der peripheren Gefässcontraction. Die erwähnten Untersuchungsergebnisse zeigen also, dass die von uns beschriebene vasomotorische Wirkung des Thyreoideapresssaftes mit dessen blutdrucksedennker Wirkung trotz dem scheinbaren Gegensatze gut in Einklang gebracht werden kann.

Von den übrigen Organen haben wir Untersuchungen mit den Presssäften von Thymus, Leber, Niere, Muskel, Milz und Gehirn angestellt. Unter diesen erwiesen sich die Presssäfte der Milz und der Muskeln als ganz wirkungslos. Der Nierenpresssaft hat nach einer kleinen Blutdrucksenkung Blutdrucksteigerung hervorgebracht.

Die Presssäfte der Leber, des Thymus und Gehirns hatten eine blutdrucksenkende Wirkung. Nach der Injection von Leberpresssaft trat eine ganz geringe und kurzdauernde Blutdrucksenkung ein, bei welcher wir auch ein kleines Anwachsen der systolischen Erhebungen beobachteten. Die Blutdrucksenkung tritt anscheinend auch zufolge einer Gefässentspannung ein, diese ist aber sehr geringfügig und die excessiv grossen systolischen Erhebungen mit hochgradigem Dicrotismus und mit allen Zeichen der grossen Gefässentspannung und mit starker Blotdrucksenkung, welche schon nach der Injection einer kleinen Dosis von Pankreaspresssalt erscheinen, zeigten sich selbst nach der Injection von sehr grossen Dosen Leberpresssaft, ja auch, wenn die Injection mehrmals wiederholt wurde, nie. Aehnlich erging es uns mit dem Gehirnpresssaft, wo wir noch erwähnen möchten, dass die durch den Presssaft verursachte Blutdrucksenkung eine hochgradige und langdauernde Arhythmie begleitete. Die durch den Thymuspresssaft verursachte Blutdrucksenkung hat ein ganz anderes Bild als die durch den Pankreaspresssaft verursachte. In der Thymuscurve ist kein einziges Zeichen einer Gefässentspannung aufzufinden, die systolischen Erhebungen werden noch kleiner und die Curve sieht eher etwas der Thyreoideacurve ähnlich.

Von diesen Versuchen ergiebt sich also, dass die Presssäfte mehrerer Organe auf den Blutdruck herabsetzend wirken. Diese Wirkungen zeigen aber einen sehr ausgeprägten Unterschied gegenüber den Wirkungen des Pankreaspresssaftes und zwar sowohl in Bezug auf den Mechanismus wie auch der Intensität und somit der Bedeutung der Wirkung. Nach der Untersuchung sämmtlicher erwähnten Presssäfte können wir sagen, dass die gefässentspannende Wirkung des Pankreaspressaftes eine ebenso specifische ist wie die vasoconstrictorische und blutdruckerhöhende Wirkung des Adrenalins, trotzdem auch Presssäfte anderer Organe in einem gewissen Grade blutdrucksteigernd wirken.

In Anbetracht dieser antagonistischen Gefässwirkung des Adrenalins und des Pankreaspresssaftes kann die Frage aufgeworfen werden, ob das Pankreas ähnlich der Nebenniere nicht eine Rolle im physiologischen Gleichgewicht des Blutkreislaufes spiele, ob nicht das Pankreas dasjenige Organ ist, dessen innere Secretion gegenüber der den sympathischen Tonus steigernden Wirkung des Adrenalins den Tonus des autonomen Nervenystems auf einer gewissen Höhe hält und den normalen Zustand der Gefässe und so des Blutkreislaufes sichert. 
340 R. Bálint u. B. Molnár, Einfluss des Pankreas-Presssaftes auf d. Blutkreislauf.

Besonders interessant ist das Verhalten der Gefässe bei Patienten, die an Morbus Basedow leiden. Wenn man bedenkt, dass bei Basedowkranken Leichen einer Adrenalinämie oder einer durch $0^{\prime}$ Connor gefundenen gesteigerten Adrenalinempfindlichkeit des sympathischen Nervensystems vorhanden sind, sollte man einen gespannten Puls und eine Blutdrucksteigerung erwarten, während in der Mehrzahl der Fälle trotz der Tachycardie und des gesteigerten Schlagvolums der Blutdruck nicht hoch zu sein pflegt, ja sogar Gefässentspannung und nicht gerade selten Dikrotie zu beobachten sind. In diesen Fällen liegt die Annahme nahe, dass die Schlaffheit der Gefässe jener Blutdrucksteigerung vorbeugt, welche durch die erwähnte Alteration der Herzthätigkeit (Tachycardie + nicht vermindertes Schlagvolum) entstehen könnte. Unsere zahlreichen klinischen Beobachtungen zeigten, dass ein Pulsus celer hauptsächlich in jenen Fällen aufzufinden ist, wo die Tachycardie eine grössere ist. Obgleich entscheidende Beweise fehlen, scheint die auffällige Thatsache, dass der Puls beim Basedow trotz des Hyperthyreoidismus und trotz der erhöhten Adrenalinempfindlichkeit oft in ausgesprochener Weise entspannt erscheint, darauf hinzuweisen, dass als ein dritter Factor eine erhöhte Pankreaswirkung die Eigenschaften des Kreislaufes bei der Basedow'schen Krankheit bestimmt. 